\title{
Assessment of Ultrasound -Guided Core Needle Biopsy as the First- Line Diagnostic Procedure for Cervical Lymphoma
}

\author{
Original Farrokh Heidari', Firouzeh Heidari', Parnian Khamushian', Farzad Yazdani \\ Article Kayvan Aghazadeh ${ }^{1}$, Ebrahim Karimi ${ }^{1^{*}}$ \\ ${ }^{I}$ Otorhinolaryngology Research Center, ${ }^{2}$ Oncology Research Center, ${ }^{3}$ Pathology Center, \\ Tehran University of Medical Sciences, Tehran, Iran.
}

\begin{abstract}
Objective: This study was conducted to assess the effectiveness of Ultrasound-guided Core Needle Biopsy (US-CNB) to provide sufficient information for diagnosis and initiation of the treatment for head and neck lymphoma.

Study Design: We carried out the prospective consecutive case series in a single- center study.

Setting: The tertiary medical center setting.

Patients and Methods: Totally, 72 patients were enrolled and the US-CNB of suspicious cervical lymph node was performed. Demographics, imaging and pathological data were collected for each patient and used to identify the factors that shape the diagnostic yield of US-CNB.

Results: We observed among 72 CNBs 52 were fully diagnosed and 20 cases needed Excisional Surgical Biopsy for subclassification. Then the factors influencing the results of the US-CNB, as the first-line diagnostic procedure for suspected cervical lymphadenopathy were discussed. The CNB results did not differ in terms of BMI, neck circumference and pathological subtype. Fully diagnosis CNB results demonstrated an association with higher mean tumor size and LAP in cervical zones 1 to 4 rather than posterior triangle.

Conclusion: About $30 \%$ of the patients in the present study required ESB after US-CNB for fully diagnosis and this delayed their treatment. Therefore, the use of US-CNB as a primary method in assessment of cervical lymphadenopathy still needs more studies to investigate the factors influencing the results and shaping its diagnostic yield.
\end{abstract}

Key Words: Cervical lymphadenopathy, core needle biopsy, Excisional surgical biopsy, Fine needle aspiration, Neck lymphoma.

Received: 18 June 2020, Accepted: 21 August 2020

Corresponding Author: Ebrahm Karimi, MD, Otorhinolaryngology Research Center, Tehran University of Medical Sciences, Tehran, Iran, Tel.: 0982166760269, E-mail: karimient@gmail.com

ISSN: 2090-0740, 2021 Vol.22

\section{INTRODUCTION}

Enlargement of head and neck lymph nodes is a common presentation of many pathological processes. The cervical lymphadenopathy is the first presentation in $70-75 \%$ of head and neck lymphoid neoplasm cases. In this regard, specific diagnosis and differentiation between benign and malignant diseases is essential. ${ }^{[1,2]}$

The 2016 World Health Organization (WHO) classification of lymphoid neoplasm represents established guidelines for diagnosis of malignant lymphomas based on clinical, pathological, and genetic or molecular data characteristics. ${ }^{[3,4]}$ Both pathologists and oncologists consider Excisional Surgical Biopsy (ESB) as the gold standard for diagnosis of lymphoma based on sufficient histologic pathological material obtained from surgically excised specimens.

In the recent years, reliance on Fine Needle Aspiration Cytology (FNAC) and Core Needle Biopsy (CNB) in evaluation of cervical lymphadenopathy when lymphoma is highly suspected has increased due to their excellent diagnostic outcomes. Many institutions have performed FNA and CNB as primary diagnostic procedures for the patients with suspected lymphoma.$^{[5-10]} \mathrm{CNB}$ is a simple, cost-effective, and outpatient procedure with minimal complications compared to ESB. In addition, CNB unlike FNA provides sufficient lymph node tissue for Immunohistochemistry (IHC) and subgroup diagnosis of lymphoma that allows the clinicians to initiate the treatment. However, the oncologists frequently question whether any image-guided biopsy techniques (FNA and $\mathrm{CNB}$ ) rather than the ESB are adequate to allow instigation of the treatment. ${ }^{[11,12]}$

To assess the effectiveness of Ultrasound-guided Core Needle Biopsy (US-CNB) to provide sufficient information for initiation of the treatment of lymphoid neoplasm, we performed the prospective consecutive case series in a 
single- center study to address: (1) how frequently USCNB results provide a specific WHO classification of lymphoma, (2) clinical, radiological, and pathological factors influencing diagnostic accuracy of the US-CNB for head and neck lymphoma.

\section{PATIENTS AND METHODS:}

This study was carried out with a prospective consecutive case series design in a tertiary medical center setting catering to both referred patients and the patients from the community. In our center, when the clinicians had diagnostic suspicion to lymphoma, according to history, physical examination, feature of mass imaging and FNA results, the patients referred for excisional biopsy (ESB). In this study, the CNB was performed for the patients before ESB, as an initial investigation. The study protocol was approved by the local Ethics Review Committee of our institution (Approval ID: IR.TUMS.MEDICINE. REC.1397.731) and written informed consent was obtained from all the patients.

Patients with the signs and symptoms of acute febrile illness and upper respiratory infection, apparent benign mass, salivary gland and thyroid tumor (history or clinical diagnostic suspicion), other primary focused head and neck malignancies, known lymphoma, transplant recipients, or those with a history of chemoradiotherapy or HIV were excluded from the study. There were no exclusions based on age, gender, or cervical lymphadenopathy (LAP) size. Totally, 72 patients were enrolled in the study from April 2016 to April 2018.

The US-CNB of cervical lymph node was taken after complete head and neck examinations and color Doppler sonography by an experienced head and neck surgeon. Each specimen was visually inspected, and multiple biopsies were collected until the surgeon was satisfied with the size of retrieved specimen. A coagulation screen was not routinely performed, and if the patient was taking Aspirin (ASA), then it continued before and after the USCNB without any changes. The US-CNB was performed by a free hand technique using the Super Core ${ }^{\mathrm{TM}}$ SemiAutomatic Biopsy Instrument $(16 \mathrm{G} \times 10 \mathrm{~cm})$ under local anesthesia with $1 \%$ lidocaine in outpatient settings. The patients were monitored for $30 \mathrm{~min}$ after the US-CNB, and gentle compression was applied on biopsy site.

All the CNBs were fixed in AFA (75\% alcohol, $0.8 \%$ formalin, and $5 \%$ acetic acid). The CNBs were evaluated by an experienced board-certified pathologist in our institution. When the biopsies were diagnosed as malignant lymphomas, a panel of antibodies and Immunohistochemistry (IHC) was performed to determine histological subtype. A subtype-specific classification of lymphoma was made according to the 2016 revision of the WHO classification of lymphoid neoplasm. ${ }^{[3,4]}$
The following terminology was used based on the suggestions by Burke et al and Skelton et al for grading diagnostic quality of the FNA and CNB specimens, which was as follows: Grade 0 (Inadequate): insufficient material for diagnosis, Grade 1 (Equivocal): pathologist was unable to differentiate reactive lymph nodes from lymphoma, Grade 2 (Partially diagnostic): consistent with lymphoma but no sub - classification was determined for the tumor, and Grade 3 (Fully diagnostic): sufficient information obtained for sub classification of lymphoma to allow instigation of the treatment. In this study, Grades 0 and 1 were considered as non-diagnostic. Partially and non-diagnostic patients underwent the ESB for further tissue harvesting , and fully diagnosed patients referred to a chemotherapist for initiation of the treatment.

Demographic data including age, gender, BMI, neck circumference, and tumor size (the largest LAP diameter obtained by ultrasound examinations), and location, and pathologic data including the results of FNA, CNB, and ESB (if done) were collected for each patient. Sensitivity, specificity, Positive and Negative Predictive Value (PPV, NPV) were calculated for CNBs in diagnosis of lymphoma. In addition, adequacy of CNBs was evaluated for a complete diagnosis and sub -classification of lymphoma and effective factors. The statistical package IBM SPSS software for Windows (version 24.0 Armonk, NY: IBM Corp) was used for data analysis. $\mathrm{T}$-test and Chi -Square test were used to compare the results obtained from assessment of CNBs between the groups in terms of continuous and categorical variables, respectively. $\mathrm{P}$-values of $<0.05$ were considered as statistically significant.

\section{RESULTS:}

Totally, 72 CNBs of cervical LAP were obtained from 72 patients (Table 1). Mean age of the patients including 45 males and 27 females was equal to 48.92 years old with an age range of 11 - 86 years old. Tumor size ranged from 1.5 to $7 \mathrm{~cm}$ with a mean of $4.37 \mathrm{~cm}$. FNA results were nondiagnostic in $45(62.5 \%)$ cases and partially - diagnostic in $27(37.5 \%)$ cases.

Among 72 CNBs, six non-diagnostic cases were reported. The ESB results for these six CNBs were two Hodgkin Lymphomas (HL), two Follicular Non-Hodgkin Lymphomas (FNHL), one Diffuse Large B -cell Lymphoma (DLBL), and one Tuberculosis (TB).

There were 59 true positive and seven true negative diagnoses. There were also one false positive and five false negative findings. Therefore, calculated sensitivity for CNB in diagnosis of lymphoma was equal to $92 \%$ in clinically suspected patients and the specificity was calculated as $87.5 \%$ (Table 2). NPV, PPV, and accuracy for CNB in diagnosis of lymphoma were calculated as 59, 98, and $92 \%$, respectively. 
Among 72 patients, eight cases were reactive lymphoma or Tuberculosis, and 64 patients were finally diagnosed with lymphoma (CNBs or ESBs). Forty-five CNB cases were fully diagnosed as $22 \mathrm{HLs}, 21$ DLBLs, and 2 Burkitt lymphomas. Fourteen and five cases were partially and non-diagnostic, respectively. Their ESB results were compatible with 11 HLs, 4 DLBLs, and 4 FNHLs (Chart 1).

Following analysis of the results, CNB cases were divided into two subgroups as positive for initiation of the treatment (fully diagnosis) and negative for initiation of the treatment (partially and non-diagnostic) that referred to the surgery. Two subgroups were compared according to BMI, neck circumference, pathological lymphoma subtype, tumor size, and Lymph Node (LN) location (Table 3). The $\mathrm{CNB}$ cases did not differ in terms of BMI ( $p$-value $=0.46)$ and neck circumference $(p$-value $=0.37)$. Regarding pathological lymphoma subtype, the two subgroups did not demonstrate any statistically difference $(p$-value $=0.35)$. The positive subgroup demonstrated a higher mean tumor size than the negative subgroup (4.65 vs. 3.54 $\mathrm{cm}, p$-value $=0.004)$. More negative CNB results in the subgroups belonged to LAP in cervical zone 5 (posterior triangle) rather than zones 1 to 4 , ( $p$-value $<0.001)$.

Table 1: Demographic parameters of the study population

\begin{tabular}{ccc}
\hline Gender & Male $\%(\mathrm{n})$ & $62.5 \%(45$ \\
Female $\%(\mathrm{n})$ & $37.5 \%(27)$ \\
\hline Age (year) & & $48.92(11-86)$ \\
BMI $\left(\mathrm{Kg} / \mathrm{m}^{2}\right)$ & $24.05(19.38-33.29$ \\
Neck circumference $(\mathrm{cm})$ & $34.4(31.7-38.6)$ \\
Mass size $(\mathrm{cm})$ & $4.37(1.50-7.00)$ \\
& $29.2 \%(21)$ \\
FNA $\%(\mathrm{n})$ & $33.3 \%(24)$ \\
& & $37.5 \%(27)$ \\
& & $0 \%(0)$ \\
\hline
\end{tabular}

Table 2: seventy-two lesions identified as positive or negative for lymphoma by core needle biopsy (CNB) pathology with subsequent comparison with surgical excision pathology or improvement after treatment as a diagnostic gold standard standard.

\begin{tabular}{lllll}
\hline & & diagnostic gold standard & & total \\
\hline CNB & positive & negative & 60 \\
& Positive & 59 & 1 & 12 \\
& Negative & 5 & 7 & 72 \\
\hline
\end{tabular}

Sensitivity $=$ true malignant needle biopsy results/total malignant surgical results $=92 \%$

Specificity $=$ true benign needle biopsy results/total benign surgical results $=87.5 \%$

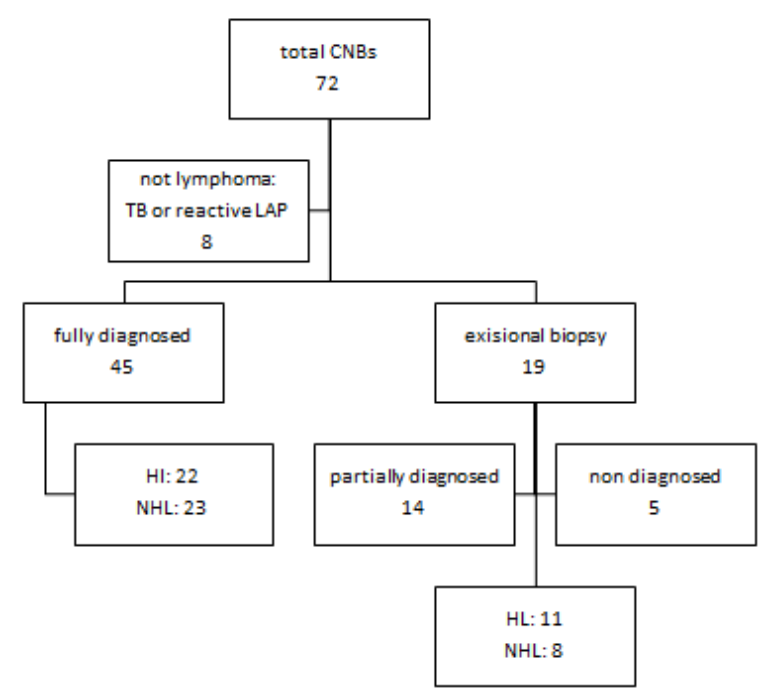

Chart 1: Core needle biopsy chart's result.

CNB: Core needle biopsy, TB: tuberculosis, LAP: lymphadenopathy, HL: Hodgkin lymphoma, NHL: non Hodgkin lymphoma. 
Table 3: Core needle biopsy result according to BMI, Neck circumference, pathological lymphoma subtype, tumor size, and lymph node (LN) location

\begin{tabular}{|c|c|c|c|c|}
\hline & \multicolumn{4}{|c|}{ Core needle biopsy result } \\
\hline & & Fully diagnosed & $\begin{array}{l}\text { Partially or non } \\
\text { diagnosed }\end{array}$ & P-value \\
\hline BMI & & 24.29 & 23.71 & 0.46 \\
\hline Neck circumference $(\mathrm{cm})$ & & 34.2 & 34.7 & 0.37 \\
\hline \multirow[t]{2}{*}{ lymphoma subtype } & Hodgkin lymphoma & 22 & 11 & \multirow{2}{*}{0.35} \\
\hline & Non Hodgkin lymphoma & 23 & 8 & \\
\hline \multirow[t]{2}{*}{ Tumor size (cm) } & & 4.65 & 3.54 & 0.004 \\
\hline & Zone 1 & 16 & 2 & \\
\hline \multirow[t]{2}{*}{ LN location } & Zone 2 to 4 & 29 & 11 & $<0.001$ \\
\hline & Zone 5 & 0 & 6 & \\
\hline
\end{tabular}

\section{DISCUSSION}

Selection of image -guided techniques (FNA and CNB) or ESB for cervical lymphadenopathy tissue sampling is influenced by numerous considerations, such as reliability, accuracy, morbidity, and costeffectiveness. ${ }^{[14]}$

Some studies have suggested the use of US-CNB as the first-line diagnostic procedure for identified and differentiated malignant lymphoma from other lymphadenopathy etiologies with an overall accuracy more than $85 \%$. This suggestion has been supported in a Randomized Clinical Trial (RCT) conducted by N. Pugliese et al (2017) who compared power Doppler US-CNB with ESB in terms of the ability to diagnose lymphoma. They showed that sensitivity of US-CNB for detection of lymphoma was equal to $98.8 \%$ (95\% $\mathrm{CI}=95.9-99.9 \%)$. They also estimated that cost per biopsy for the US-CNB was 24-fold lower compared to standard ESB. ${ }^{[15]}$ Adding these observations to the fact that, US-CNB reduces the patients' psychological and physical pain as well as risk of surgery and hospitalization, the use of US-CNB is recommended under optimal study conditions for both superficial and deep lymphadenopathy as the first-line diagnostic procedure for patients with a suspected lymphoma.

Despite the results of the recent studies, US-CNB seems a safe and efficient procedure for diagnosing the cause of cervical lymphadenopathy; and clinical guidelines (European Society of Medical Oncologists (ESMO) and the National Comprehensive Cancer Network (NCCN)) recommend the use of ESB for an initial diagnosis and classification of hematolymphoid neoplasm due to the fact that FNAB or CNB may lack histological architecture that is necessary for fully diagnosis and to allow instigation of the treatment. ${ }^{[16-20]}$

Several studies such as a systematic review by Frederickson et al (2015) ${ }^{[6]}$ and a recent retrospective single-center cohort study in Korea ${ }^{[21]}$ show that the median rate at which CNBs yielded a subtype-fully diagnosis of lymphoma was about $70 \%$. Therefore, $30 \%$ of CNBs must be followed by administration of ESB to sub classify lymphoma and provide optimal diagnostic information for the oncologist's therapeutic options that can delay initiation of the treatment. In HL and FNHL, this rate may be worse and there may be higher need for performing the ESB to harvest sufficient material for fully diagnosis.

The results of our study are almost similar to previous studies. In our study, sensitivity, specificity, and accuracy for CNB in detecting lymphoma were calculated as $92,87.5$, and $92 \%$, respectively. Moreover, $70 \%(45 / 64)$ of CNB cases were fully diagnosed.

In the following, the factors influencing the results of the US-CNB, as the first-line diagnostic procedure for suspected cervical lymphadenopathy were identified. The CNB results were statistically different in terms of tumor size and LN location. The fully diagnosed cases demonstrated a higher mean tumor size $(4.65$ vs. $3.54 \mathrm{~cm})$ and more information was obtained from LAP in cervical zones 1 to 4 rather than zone 5 (posterior triangle) while, there were a small number of specimens at the cervical zone 5 ( 6 vs. 58 specimens).

In our study, the CNB results did not differ in terms of BMI and neck circumference. Confirmed and deferred results were also compared in terms of their pathological subgroups. However, DLBL was fully diagnosed more by performing CNB than other pathological subtypes (77\% vs. $65 \%)$ but it was not statistically significant.

\section{CONCLUSION}

US-CNB is an inexpensive, safe, and accurate procedure for diagnosis of neck lymphoma. However, about $30 \%$ of the patients in the present study 
required ESB after US-CNB for fully diagnosis and this delayed their treatment. Therefore, the use of US-CNB as a primary method in assessment of cervical lymphadenopathy still needs more studies to investigate the factors influencing the results and shaping its diagnostic yield. These factors can increase efficiency of the results and identify suspected patients who benefit more from administration of US-CNB as the first-line diagnostic procedure.

\section{LIST OF ABBREVIATION}

Ultrasound-guided Core Needle Biopsy (US-CNB), Lymphadenopathy (LAP), Core Needle Biopsy (CNB), Excisional Surgical Biopsy (ESB), Fine Needle Aspiration Cytology (FNAC), Fine Needle Aspiration (FNA), Immunohistochemistry (IHC), Human Immunodeficiency Viruses (HIV), Positive Predictive Value (PPV), Negative Predictive Value (NPV), Hodgkin Lymphomas (HL), Follicular Non-Hodgkin Lymphomas (FNHL), Diffuse Large B -cell Lymphoma (DLBL), Tuberculosis (TB), Randomized Clinical Trial (RCT).

\section{CONFLICT OF INTEREST}

There are no conflicts of interest.

\section{REFERENCES}

1. Urquhart A, Berg R. Hodgkin's and non $\square$ Hodgkin's lymphoma of the head and neck. The Laryngoscope. 2001;111(9):1565-9.

2. Cooper JS, Porter K, Mallin K, Hoffman HT, Weber RS, Ang KK, et al. National Cancer Database report on cancer of the head and neck: 10-year update. Head \& Neck: Journal for the Sciences and Specialties of the Head and Neck. 2009;31(6):748-58.

3. Arber DA, Orazi A, Hasserjian R, Thiele J, Borowitz MJ, Le Beau MM, et al. The 2016 revision to the World Health Organization classification of myeloid neoplasms and acute leukemia. Blood. 2016;127(20):2391-405.

4. Swerdlow SH, Campo E, Pileri SA, Harris NL, Stein $\mathrm{H}$, Siebert R, et al. The 2016 revision of the World Health Organization classification of lymphoid neoplasms. Blood. 2016;127(20):2375-90.

5. Novoa E, Gürtler N, Arnoux A, Kraft M. Role of ultrasound $\square$ guided core $\square$ needle biopsy in the assessment of head and neck lesions: a meta $\square$ analysis and systematic review of the literature. Head \& neck. 2012;34(10):1497-503.

6. Frederiksen JK, Sharma M, Casulo C, Burack WR. Systematic review of the effectiveness of fine-needle aspiration and/or core needle biopsy for subclassifying lymphoma. Archives of Pathology and Laboratory Medicine. 2015;139(2):245-51.

7. Ryu YJ, Cha W, Jeong WJ, Choi SI, Ahn SH Diagnostic role of core needle biopsy in cervical lymphadenopathy. Head \& neck. 2015;37(2):229-33.

8. Skelton E, Jewison A, Okpaluba C, Sallomi J, Lowe $\mathrm{J}$, Ramesar K, et al. Image-guided core needle biopsy in the diagnosis of malignant lymphoma. European Journal of Surgical Oncology (EJSO). 2015;41(7):8528.

9. Assaf N, Nassif S, Tamim H, Bazarbachi A, Zaatari G, Chakhachiro Z. Diagnosing Lymphoproliferative Disorders Using Core Needle Biopsy Versus Surgical Excision with Limited Resources-A Three-year Experience of a Reference Center in Lebanon. Clinical Lymphoma Myeloma and Leukemia. 2019.

10. Jelloul F-Z, Navarro M, Navale P, Hagan T, Cocker RS, Das K, et al. Diagnosis of Lymphoma Using FineNeedle Aspiration Biopsy and Core-Needle Biopsy: A Single-Institution Experience. Acta cytologica. 2019;63(3):198-205.

11. Kim BM, Kim E-K, Kim MJ, Yang W-I, Park CS, Park SI. Sonographically guided core needle biopsy of cervical lymphadenopathy in patients without known malignancy. Journal of ultrasound in medicine. 2007;26(5):585-91.

12. Pfeiffer J, Kayser G, Technau $\square$ Ihling K, Boedeker CC, Ridder GJ. Ultrasound $\square$ guided core $\square$ needle biopsy in the diagnosis of head and neck masses: Indications, technique, and results. Head \& Neck: Journal for the Sciences and Specialties of the Head and Neck. 2007;29(11):1033-40.

13. Burke $\mathrm{C}$, Thomas $\mathrm{R}$, Inglis $\mathrm{C}$, Baldwin A, Ramesar $\mathrm{K}$, Grace $\mathrm{R}$, et al. Ultrasound-guided core biopsy in the diagnosis of lymphoma of the head and neck. A 9 year experience. The British journal of radiology. 2011;84(1004):727-32.

14. Allin D, David S, Jacob A, Mir N, Giles A, Gibbins $\mathrm{N}$. Use of core biopsy in diagnosing cervical lymphadenopathy: a viable alternative to surgical excisional biopsy of lymph nodes? The Annals of The Royal College of Surgeons of England. 2017;99(3):242-4.

15. Pugliese N, Di Perna M, Cozzolino I, Ciancia G, Pettinato G, Zeppa P, et al. Randomized comparison of power Doppler ultrasonography-guided coreneedle biopsy with open surgical biopsy for the 
characterization of lymphadenopathies in patients with suspected lymphoma. Annals of hematology. 2017;96(4):627-37.

16. Tilly H, Gomes da Silva M, Vitolo U, Jack A, Meignan M, Lopez-Guillermo A, et al. Diffuse large B-cell lymphoma (DLBCL): ESMO Clinical Practice Guidelines for diagnosis, treatment and follow-up. Annals of oncology. 2015;26(suppl_5):v116-v25.

17. Dreyling M, Ghielmini M, Rule S, Salles G, Vitolo U, Ladetto M. Newly diagnosed and relapsed follicular lymphoma: ESMO Clinical Practice Guidelines for diagnosis, treatment and follow-up. Annals of Oncology. 2016;27(suppl_5):v83-v90.

18. Hoppe RT, Advani RH, Ai WZ, Ambinder RF, Aoun P, Bello CM, et al. Hodgkin lymphoma version 1.2017, NCCN clinical practice guidelines in oncology. Journal of the National Comprehensive Cancer Network. 2017;15(5):608-38.
19. Eichenauer D, Aleman B, André M, Federico M, Hutchings $\mathrm{M}$, Illidge $\mathrm{T}$, et al. Hodgkin lymphoma: ESMO Clinical Practice Guidelines for diagnosis, treatment and follow-up. Annals of Oncology. 2018;29:iv19-iv29.

20. Chaganti S, Illidge T, Barrington S, Mckay P, Linton $\mathrm{K}$, Cwynarski $\mathrm{K}$, et al. Guidelines for the management of diffuse large $\mathrm{B} \square$ cell lymphoma. British journal of haematology. 2016;174(1):43-56.

21. Kwon M, Yim C, Baek HJ, Lee JS, Seo J-H, Kim $\mathrm{JP}$, et al. Ultrasonography-guided core needle biopsy of cervical lymph nodes for diagnosing head and neck lymphoma compared with open surgical biopsy: exploration for factors that shape diagnostic yield. American journal of otolaryngology. 2018; 39(6):679-84. 